\title{
Conceptos claves para la articulación entre Vida y Ser en la ontología de Gilles Deleuze
}

\author{
Key concepts for the articulation between Life and Being in the \\ ontology of Gilles Deleuze.
}

\author{
Celeste Florencia Ramírez \\ Escuela de Humanidades / Universidad Nacional de San Martín (ARGENTINA) \\ CE: ramirezcelestef@hotmail.com ID ORCID: 0000-0001-9351-0154
}

DOI: $10.32870 /$ sincronia.axxiii.n76.9b19

(C) $\mathrm{BY} \cdot \mathrm{NC}$

Esta obra está bajo una Licencia Creative Commons Atribución-NoComercial 4.0 Internacional

Recibido: $25 / 03 / 2019$

Revisado: 02/04/2019

Aprobado: 20/05/2019

\section{RESUMEN}

El presente trabajo tiene como objetivo establecer, en líneas generales, cuál es la concepción que tiene Deleuze sobre la vida. Para esto, recurrimos a la lectura de la clase del 14 de enero de 1974, dictada por Deleuze en la Universidad de Vincennes y la clase del 24 de marzo de 1981, pronunciada por él también en la misma universidad. En estas clases Deleuze introduce una proposición que se extiende a lo largo de la Edad Media, incluido el siglo XVII, la cual plantea un problema relacionado a la naturaleza del Ser. Al respecto, intentaremos establecer cuál fue el origen de dicha noción en los escritos de Spinoza. Si bien Deleuze renuncia al abordaje profundo de las tradiciones filosóficas sobre el tema, realizaremos una breve introducción sobre las perspectivas equivocista, univocista y análoga, para mostrar que Spinoza descubre, como considera Deleuze (2008, p.493), una nueva esfera del Ser. La ontología spinozeana aparecerá aquí como la única en sentido riguroso (Deleuze, 2008, pp.69 y 483). 
A lo largo de este trabajo se pretenderá dar cuenta de los múltiples elementos que conforman a la vida como plano de inmanencia. Al respecto, intentaremos corroborar que este plano de inmanencia se identifica necesariamente con una Ética.

Palabras clave: Ontología. Univocidad. Etología. Plano de inmanencia. Vida.

\begin{abstract}
:
The objective of this paper is to establish, in general terms, what Deleuze's conception of life is, mainly by reading the class of January 14th 1974 , dictated by Deleuze at the University of Vincennes and the class of March 24th 1981, pronounced by him also at the same university. In these classes Deleuze introduces a proposition that extends throughout the Middle Ages, including the seventeenth century, which poses a problem regarding the nature of Being. In this regard, we will try to establish what was the origin of this notion in the writings of Spinoza. Although Deleuze rejects the deep approach of the philosophical traditions on the subject, we will make a brief introduction about the unjust, univocal and analogous perspectives, to show that Spinoza discovers, as Deleuze (2008, p.493) considers, a new sphere of Being. The Spinozean ontology will appear here as the only one in a rigorous sense (Deleuze, 2008, pp.69, 483).

Throughout this work we will try to account for the multiple elements that make up life as a plane of immanence. In this regard, we will try to corroborate that this plane of immanence is necessarily identified with an Ethics.
\end{abstract}

Keywords: Ontology. Univocity. Ethology. Plane of immanence. Life.

\title{
La potencia infinita del Ser
}

El enorme problema que intentaremos dilucidar está en la noción de hen panta (Deleuze, 2008, p.484). Se trata de un término griego, es decir, que se encuentra en el origen de la filosofía misma, a saber: hen panta. Hen quiere decir "lo uno"; panta significa "todas las cosas", se trata de un nominativo plural neutro. La traducción completa sería "lo uno todas las cosas". De allí que la filosofía ha tenido un vínculo con el pananteísmo: pan quiere decir "todo", an quiere decir "uno", 
teísmo, Teos, "Dios". Por lo tanto, panteísmo o pananteísmo quiere decir "lo uno en todas las cosas", esto es "Dios". Si uno se pregunta acerca de qué es aquello que constituye la esencia de una cosa cualquiera, seguramente imaginaríamos que tiene que Ser algo único, no puede Ser algo disperso y múltiple. El problema está en que esa esencia, propia de una realidad particular, lo que hace que un caballo sea caballo, si cada uno de los caballos tiene una esencia, entonces, a cada uno debería corresponderle una palabra distinta, y, sin embargo, es la misma: "caballo". La pregunta sería: ¿Cuál es la relación entre algo que se nos tiene uno y que, sin embargo, se refiere a una multiplicidad de individuos? Más precisamente, ¿cuál es el vínculo de una forma finita con la forma infinita? "enorme problema que va a animar toda la teología" (Deleuze, 2008, p.486).

Deleuze afirma que únicamente Spinoza ha logrado una ontología en un sentido riguroso, puesto que extrema las posibilidades de la proposición hen panta. Esto lo logra, principalmente, en la siguiente afirmación: "las mismas formas, o si prefieren, los mismos atributos [...] se dicen de Dios y de las cosas" (Deleuze, 2008, p.485). En esta proposición podemos dar cuenta de la pertenencia del filósofo Baruch de Spinoza a la marginalidad, marcada por el rechazo tanto al cristianismo como al judaísmo, del cual procedía, y que, hacia 1656, le valdrá su Cherem (Deleuze, 2004, p.19). Pero, ¿cómo expresar con palabras el coraje de un filósofo que rompe a sabiendas con las dos instituciones que regían en su tiempo? Y es que dicha proposición, que para la gran mayoría del resto de los filósofos es una herejía, más aún, explicita Deleuze, una blasfemia, presenta una novedad, a saber: las mismas formas infinitas que se atribuyen a Dios, se las atribuye bajo la misma forma a las criaturas. Es decir, hay formas comunes a la substancia y a los entes (Deleuze, 2008, p.486).

Si queremos comprender esta primera parte de la ontología spinozeana, tendremos que centrarnos en dilucidar ciertas nociones: equivocidad, univocidad, analogía. La cuestión sería: ¿existen formas que se atribuyen tanto al Ser infinito como a los seres finitos, a Dios y a las criaturas? 
La equivocidad implica sostener que el término Ser se enuncia en varios sentidos de eso de lo que se dice, siendo que, aquellos muchos sentidos de la palabra Ser no tienen medida común, es decir, tienen un sentido totalmente diferente (Deleuze, 2005, p.282).

Sin embargo, también están aquellos que consideran que el Ser es análogo. Santo Tomás de Aquino, quien tiene una célebre teoría de la analogía del Ser, sostenía que el Ser se enuncia en muchos sentidos de aquello que se dice y considera, a diferencia de los partidarios de la equivocidad, que la medida en común está regida por relaciones de analogía, es decir que se dice de Dios y de las criaturas de manera analógica (Deleuze, 2008, p.491).

Ahora bien, Santo Tomás distinguía dos tipos de analogías científicas o técnicas: por un lado, la analogía de proporción o serie y, por el otro, la analogía de proporcionalidad o estructura.

La primera considera que el Ser se dice en varios sentidos y que esos sentidos tienen una medida en el concepto. De esta manera, hay un primer sentido de la palabra Ser y luego sentidos diferentes que derivan, siguiendo una regla de proporción del primer sentido. Según esta analogía, sería: A es semejante a B, B es semejante a $C$, donde $A$ sería un supuesto término superior como razón de la serie, ya sea por su cualidad o perfección, y luego le siguen todos esos términos vinculados al primero según sus graduaciones. La palabra Ser tenía como primer sentido el término "substancia" o "esencia". Por lo tanto, la substancia también tenía analogías. A fin de cuentas, la analogía de proporción consiste en jerarquizar y ordenar una pluralidad de sentidos de un primer sentido del término Ser.

La segunda implicaba una analogía matemática: A es semejante a B lo que C es semejante a D. Por ejemplo, la justicia finita es a las cosas finitas lo que la justicia infinita es al Ser infinito. El Ser infinito se enuncia de Dios, en cambio, el Ser finito se dice de las criaturas. Es decir, Dios posee en sí la justicia como cualidad plena, en cambio, el hombre tiene por derivación la justicia de manera secundaria.

En ambas analogías, Deleuze $(2006$, p.241) marca que la naturaleza es entendida bajo la forma de mimesis: la primera bajo la forma de criaturas que, por semejanza graduada, imitan un 
único termino eminentemente divino que tienen como modelo; la segunda bajo la forma de un modelo en espejo que también imitan, pero por diferencia graduada.

Dicha noción de analogía estaba fuertemente vinculada a la noción de moral, es más, son inseparables la una de la otra. Y es que la esencia y los valores se encuentran íntimamente relacionadas con la moral, ya que "Una moral es una operación que nos devuelve la esencia, es decir, nuestra esencia, y que lo hace a través de los valores" (Deleuze, 2008, p.70). En este sentido, la esencia no se encuentra realizada por sí misma, sino que está en potencia en el hombre. La moral sería el medio por el cual la esencia humana se realiza. De esta manera, necesariamente, por medio de la moral, los seres humanos deben considerar la esencia del hombre como un fin.

Para la moral, la esencia del hombre se basa en la definición de Aristóteles: el hombre es un animal racional. Sin embargo, Deleuze (2008) afirma: "Toda la concepción clásica del hombre consiste en invitarlo a reunirse con su esencia, porque esa esencia es como una potencialidad que no está necesariamente realizada" (p.71). A fin de cuentas, si la esencia del hombre aún no está concretada, entonces, el objetivo de la moral es hacer pasar la esencia de potencia al acto.

Asimismo, Deleuze niega cualquier posibilidad de realizar una ontología de la moral, puesto que la moral se vale de una instancia superior al Ser, necesita una trascendencia para juzgar. De esta manera, el sistema de juicio toma como evidencia los valores.

En contraposición a este planteo, Deleuze introduce a Duns Scoto, quien posiciona el Ser en la univocidad. El Ser se dice en un único y mismo sentido de todo aquello de lo que se enuncia. Para Duns Scoto, el Ser no es análogo, sino unívoco, ya que define al Ser como lo indiferente a lo finito y a lo infinito, es decir, el Ser en tanto que Ser. Se trata de un Ser neutro, ni lo uno ni lo otro (Deleuze, 2008, p.492). En definitiva, no hay más que un único y mismo sentido de la palabra Ser. Ahora bien, el Ser se encuentra vinculado con Dios y las criaturas de forma análoga, ya que estos no son lo mismo. "Diciendo esto, creía protegerse contra el panteísmo" (Deleuze, 2008, p.491). A fin de cuentas, el Ser en tanto que Ser es unívoco metafísicamente, pero, el Ser se enuncia en muchos sentidos, fisicamente (Deleuze, 2005, p.285). 
Estas distinciones son retomadas por Spinoza, quien considera que "todas las formas que pueden Ser atribuidas a Dios, se las atribuyen también bajo la misma forma al hombre" (Deleuze, 2008, p.486). Las formas comunes que Spinoza encuentra son los atributos de la extensión y el pensamiento.

Como vemos, a Spinoza no le resulta ajena dicha problemática. Por el contrario, advertimos cómo Spinoza se encarga de hacer especial hincapié en la incidencia de la univocidad de la substancia. Según sus afirmaciones, la naturaleza es una unidad que se expresa en la diversidad de los infinitos atributos, los cuales constituyen su esencia, su existencia. Se trata del vínculo entre lo Uno y lo Múltiple.

El gran problema es que aquello en lo que no difieren es potencialmente infinito. No obstante, la naturaleza de la substancia no expresa la infinitud, inmutabilidad o la eternidad, puesto que son las propiedades de la misma. En otras palabras, las propiedades no son atributos, por lo tanto, no forman la esencia del Ser:

no es a través de atributos humanos (bondad, justicia, caridad...) que Dios comunica a las criaturas humanas las perfecciones que poseen. Al contrario, es a través de sus propios atributos que Dios comunica a todas las criaturas la potencia que le es propia. (Deleuze, 1999, p.84).

Entonces, si hay un único Ser, ¿cuál es la diferencia entre las criaturas? Desde esta perspectiva, los entes se definen por grados de potencia.

\section{La ética ontológica}

En la proposición III y su demostración, Spinoza (2004) postula:

No puede una cosa Ser causa de otra, si entre sí nada tienen en común. Si nada común tienen una con otra, entonces no pueden entenderse una por otra, $y$, por lo tanto, una no puede Ser causa de la otra. (p.58). 
Allí está afirmando que efectivamente existe algo idéntico y presente en ambos. Aquella forma común son los atributos, es decir, verbos que expresan cualidades ilimitadas que percibimos de Dios como constitutivos de la esencia del mismo. Como explica Deleuze (1999): “Los atributos [...] no cambian de naturaleza al cambiar de sujeto, es decir, cuando se las predica del Ser infinito y de los seres finitos, de la substancia y de los modos, de Dios y de las criaturas" (p.42). En la medida en que las nociones comunes encuentran su fundamento en el estatuto del atributo, significa el carácter unívoco de los atributos; puesto que constituyen la Naturaleza naturante y contiene la naturaleza naturada que, a su vez, las vuelve a expresar. Entonces, "los mismos atributos reconocen su pertenencia a la substancia que componen y a los modos que contienen" (Deleuze, 2004, p.67). De esta manera, la expresión característica de los atributos, conlleva una relación inmanente.

De este modo, se observa que los modos tienen una potencia idéntica a su esencia formal, ya que estos últimos son las condiciones bajo las cuales se atribuye a los modos la esencia de la substancia.

Las criaturas, en tanto modos finitos, únicamente pueden concebir dos atributos infinitos: por un lado, el atributo de la extensión correspondiente al cuerpo; y por el otro, el atributo del pensamiento correspondiente al alma. En otras palabras, la constitución de las criaturas solo le permite conocer esos dos atributos, sin embargo, comprendemos que hay infinitos atributos en la substancia por su calidad de potencia absolutamente infinita, que no puede abarcar solo el pensamiento y la extensión.

Además, los modos se distinguen unos de otros, ya sea por los atributos de la substancia o por los modos, puesto que estos últimos tienen su causa en otra cosa. A su vez, las substancias se diferencian entre ellas por los atributos que las componen, puesto que son en sí mismas y se conciben por sí mismas. No podría haber dos substancias con los mismos modos, porque la substancia los produce. Tampoco podríamos considerar que dos substancias compartan un mismo atributo, ya que, por la definición de substancia, es imposible que una sea causa de otra. De esta manera, hay una misma substancia para todos los atributos (Deleuze, 1999, pp.23-30). "Pues, si la substancia pudiese Ser producida por otra cosa, su conocimiento debería depender del 
conocimiento de su causa y, por lo tanto, no sería una substancia" (Spinoza, 2004, p.42). Por esto mismo, Spinoza demuestra que el Ser es ilimitado, puesto que, como vimos, si la substancia estaría limitada, debería estarlo por otra de igual naturaleza que lo limite, o limitarse a sí misma.

En consecuencia, podríamos considerar a cada forma de la naturaleza, infinitamente perfecta. Esto se debe a que existe una igualdad entre los atributos de la substancia, ninguna forma es inferior o superior a otra. En palabras del propio Deleuze (1999): "siendo todas las formas iguales (atributos). Dios no puede poseer una sin poseer las otras; no puede poseer una que valga por otra inminentemente" (p.63). Lo propio de cada atributo implica lo infinitamente perfecto, o sea, la naturaleza de Dios. Sin embargo, es necesario aclarar que "infinito" y "perfecto" son simplemente una propiedad de la naturaleza del Ser, se trata de la manera en la que cada uno de los atributos expresa la esencia de Dios. Por esto mismo, si los atributos son las nociones generales que se encuentran en la substancia y los modos, entonces, toda la naturaleza es perfección. Este principio de igualdad en las formas de la naturaleza no conlleva ninguna carencia, no hay algo que esté en falta; y es que no hay una falta originaria.

Debemos advertir que, para definir la esencia del Ser, es necesario considerar que la modalidad del Ser está determinada por una infinidad de atributos, lo absolutamente infinito. Precisamente el carácter absoluto constituye la naturaleza de la substancia, mientras que lo infinito es lo propio de cada uno de los atributos. Deleuze (1999) explica: "Siendo todas las formas de Ser infinitamente perfectas, deben pertenecer sin limitación a Dios como a un Ser absolutamente infinito" (p.63). De esta manera, Spinoza nos invita a pasar del Infinito al Absoluto, o sea, de lo perfectamente infinito a lo infinitamente absoluto. Tal como argumenta: "Por Dios entiendo un Ser absolutamente infinito, esto es, una substancia que consta de infinitos atributos, cada uno de los cuales, expresa una esencia externa e infinita" (Spinoza, 2004, p.40). No solo hay una única substancia que los modos conciben con determinados atributos, sino que aquello que pasa en la substancia tiene que reflejarse de la misma forma en todos los aspectos bajo los cuales podemos concebirla. 
A fin de cuentas, se trata de una sola y única Naturaleza para todos los vivientes, para todos los cuerpos, para todos los individuos. Deleuze (2004) plantea: "Ya no se trata de la afirmación de una substancia única, se trata del despliegue de un plan común de inmanencia en el que son todos los cuerpos, almas, individuos" (p.149). El plano de inmanencia se compone de múltiples planos, en la medida que todos esos planos avienen uno. Sin embargo, el plano o la univocidad de sus partes, constituyen una totalidad abierta, sin límites. En efecto, se trata de la conformación de un sistema que recurre desplegarse desde la multiplicidad. Lo múltiple no solo es lo que tiene muchas partes, sino lo que está plegado de muchas maneras. Podemos apreciar como la filosofía deleuzeana propone, incluso, una nueva forma de sistema puesto que, distinto al sistema cerrado y totalizante, está permanentemente abierta la posibilidad de nuevas conexiones y desuniones (Mengue, 2008, pp.57-58). La multiplicidad se rige, justamente, por un despliegue de tipo rizomático. Un rizoma es aquella planta que se expande no desde el tallo o la raíz, sino que crece y se despliega desde el medio en direcciones cambiantes, conectando un punto cualquiera con cualquier punto, es decir, con diferentes naturalezas e incluso regímenes de signos (Deleuze, 2006, p.13)

Esta característica de todo abierto prohíbe la unificación de los planos en una forma jerarquizada o trascendente y permite la pluralidad, la expansión y la diversificación de dimensiones crecientes. Esta abertura o amplitud implica lo indeterminado, es decir, el caos. De tal modo que caos no es lo opuesto al sistema, sino que esto se explica por su rasgo fundamental: el Ser inmanente. La inmanencia es justamente aquello que no tiene ni principio ni final, es decir, no tiene un "arché", origen o fundamento primero. Por este motivo, el plano de inmanencia es un repliegue de todos los planos sobre sí, es el "centramiento" o el movimiento que se despliega desde y para sí mismo (Mengue, 2008, pp.71-73).

Veremos cómo luego el plano de inmanencia se corresponde a una vida. Y es que la realidad es la expresión de la vida misma de Dios (Deleuze, 1999, p.30). Es justamente por el plano de inmanencia que podemos afirmar que hay una igualdad de las formas del Ser. Como dijimos anteriormente, si todo atributo expresa la esencia de la substancia bajo cierto modo, ningún modo es inferior o superior a otro. Esto se debe a que los atributos son realmente distintos, lo cual no 
supone que se reduzcan los unos a los otros, ya que si cada uno se concibe por sí, entonces, no hay contradicción, son necesariamente concordantes (Deleuze, 1999, p.72). De allí que el Ser conserva de atributos diferentes toda su positividad, rechazando la división de la substancia.

De esta manera, es imposible que haya diferencia de substancia, distinción de formas, género o especie, ya que de esta forma no podría librarse de la analogía del Ser. De hecho, Spinoza niega la existencia de esencias generales como una esencia del hombre, las considera confusas. Y es que, para Spinoza, la esencia es siempre una determinación singular, la esencia de este, de aquel, de ella (Deleuze, 2008, p.72). No se trata de un ente cualquiera, es lo que ese ente en particular puede $^{1}$. Tal como expresa Deleuze (2008, p.75): "Se trata de las acciones y pasiones de las cuales algo es capaz. No lo que la cosa es, sino lo que es capaz de soportar y capaz de hacer". La potencia es idéntica a la esencia misma, "la potencia es el Ser" (Spinoza, 2004, p.73). En pocas palabras, el problema no es el de los órganos y las funciones, no es el de la organización, tampoco se trata del desarrollo o la diferenciación. El problema es el de la composición: del movimiento y el reposo, la velocidad y la lentitud (Deleuze, 2006, p.259). Los individuos se definen según la composición de la relación en la que cada una de sus partes entra, por este motivo, cada uno es una multiplicidad. La multiplicidad, entonces, se despliega en el medio. El medio es el lugar donde las cosas toman velocidad, es decir, el individuo se mide según el entrecruzamiento de múltiples fuerzas con distintas intensidades, según el grado de velocidad o el movimiento o el reposo. Deleuze (2008) aclara: "Si digo 'racional', no se trata de la esencia del hombre, sino de algo que el hombre puede. Estar loco también forma parte del poder del hombre" (p.74).

La ontología en Spinoza toma el nombre de ética, entendida como una etología. La etología es una disciplina de las maneras de Ser. Así como el estatuto de los modos es su manera de Ser en el Ser. La ética no busca juzgar, al contrario, busca "los afectos activos y pasivos de los que es capaz el animal, en un agenciamiento individuado del que forma parte" (Deleuze, 2006, p.261).

\footnotetext{
${ }^{1}$ Nicolás de Cusa había creado una palabra en latín. Se trata del término possest, conformado por dos verbos: posse, que es el infinitivo del verbo poder, y est, que es la tercera persona del presente indicativo del verbo Ser. En definitiva, possest quiere decir lo que puede en acto. Se trata de la identidad de la potencia y el acto (Cfr. Deleuze, 2008, p.76).
} 
La originalidad de Spinoza es que no hay una primacía de un modo sobre otro, ni superioridad entre las criaturas. Rechaza, como sostiene Deleuze, cualquier tipo de trascendencia; la ética solo puede comprenderse desde una substancia causal inmanente (Deleuze, 1999, p.103).

\section{Conclusión}

En resumen, hasta ahora nos hemos encargado de mostrar uno de los alcances del concepto de inmanencia expresados en la teoría spinozeana y, simultáneamente, de la manera en que Deleuze, a partir de dicha noción, postula la naturaleza del Ser es su potencia.

No obstante, para concluir es importante retomar el concepto de plano de inmanencia. Deleuze (1995) hace hincapié en que "la substancia y los modos son en la inmanencia" (p.234). En este sentido, la inmanencia se encuentra atravesada por la naturaleza de la substancia. Por este motivo, el plano de inmanencia no necesita ningún objeto o sujeto que le sea de recipiente. El plano es en sí mismo, es causa de sí, no recurre a una unidad trascendente. Este plano de inmanencia o de consistencia no se trata, entonces, de un proyecto o de una intención del espíritu, sino que "tiene un sentido geométrico, sección, intersección, diagrama" (Deleuze, 2004, p.149). Vemos como rechaza cualquier analogía. Por este motivo, Deleuze (1995) señala que "una vida es la inmanencia de la inmanencia, la inmanencia absoluta: es potencia, beatitud completa" (p.234).

Spinoza presenta dos definiciones de lo que es un cuerpo. La primera es denominada cinética y define al cuerpo como las relaciones de reposo y movimiento o de velocidad y lentitud entre infinitas partículas. No define al cuerpo por sus órganos o sus funciones, sino que la forma y las funciones que el cuerpo alcance dependerá de las relaciones cinéticas que conforme. También se define al cuerpo por su poder de Ser afectado o de afectar por otro cuerpo. Esta última definición es conocida como dinámica. Ambas constituyen la individualidad de un cuerpo. Estas proposiciones conllevan a diferentes maneras de vivir, "ya no hay forma, sino tan sólo relaciones de velocidad entre partículas ínfimas de una materia no formada. Ya no hay sujeto, sino tan sólo estados afectivos individuales de la fuerza anónima" (Deleuze, 2004, p.156). Vivientes cuyas partes se 
transforman, varían, de infinitas formas, potencias heterogeneas desplazándose en un mismo plano:

La Ética de Spinoza nada tiene que ver con la moral; Spinoza la concibe como una etología, o sea, como una composición de velocidades y de lentitudes, de poderes de afectar y de Ser afectado en este plan de inmanencia. (Deleuze, 2004, p.152).

Spinoza se refiere a que no sabemos lo que puede un cuerpo, en lo bueno y en lo malo. Se trata de otra manera de concebir la vida, ya no por una especie, sino por las relaciones que cada viviente desde su individualidad es capaz de componer. Se trata de vínculos que se acomodan en medio de - acordes a - cierto ritmo a otras cosas.

Ahora bien, una vida no solo implica las vivencias que atraviesan los vivientes, sino que la vida es impersonal, indefinida, es decir, no termina en la muerte de una vida individual. Se trata de "Una vida de pura inmanencia, neutra, más allá del bien y del mal, ya que sólo el sujeto que la encarnaba en medio de las cosas la hacía mala o buena" (Deleuze, 1995, p.235). Una vida que se encuentra en constante movimiento, cambiante, penetrada por acontecimientos, singularidades o virtualidades que la hacen fluctuar.

\section{Referencias}

Deleuze, G.; y Guattari, F. (2006). Mil Mesetas Capitalismo y esquizofrenia. Valencia: Pre-Textos.

Deleuze, G.; (1995). La inmanencia: una vida. Paris: revista "Philosophie”, Vol. VII, Nro. 47, pp.233-236.

Deleuze, G.; (1999). Spinoza y el problema de la expresión. Barcelona: Muchnik Editores.

Deleuze, G.; (2004). Spinoza: Filosofía práctica. Buenos Aires: Tusquets.

Deleuze, G.; (2005). Derrames entre el capitalismo y la esquizofrenia. Buenos Aires: Cactus.

Deleuze, G.; (2008). En medio de Spinoza. Buenos Aires: Cactus.

Mengue, P. (2008). Deleuze o el sistema de lo múltiple. Buenos Aires: Las cuarenta.

Spinoza, B. (2004). Ética: demostrada según el orden geométrico. Madrid: Editorial Nacional. Trad. Vidal Peña (1980). 